TMR5 (Zedupex ${ }^{\mathrm{TM}}$ ) is a product of a Kenyan medicinal plant, prepared as a lyophilized extract and a cream. The products have been evaluated for preclinical safety and efficacy in suitable in vitro and in vivo systems of herpes infections. Herpes is a viral infection affecting over $60 \%$ of the sub-Saharan Africa young adult population. It is caused by two similar viruses, HSV-1 and HSV-2 which share $50 \%$ gene sequence homology. The infection in a major cause of genital ulcer disease, associated with increased risks of HIV acquisition and transmission. The aim is to develop TMR5 as an alternative antiherpes agent, this being necessitated by increased resistance to available drugs and the cost of the drug of choice, acyclovir, in the region. Using the trypan blue exclusion test, plaque inhibition and viral yield reduction assays for assessment of cytotoxicity $\left(\mathrm{CC}_{50}\right)$ and efficacy $\left(\mathrm{EC}_{50}\right)$, and Mice and guinea pig cutaneous and genital HSV infection models respectively following oral and topical treatments, TMR5 exhibited no cytotoxicity in mammalian cell lines with a wide therapeutic index $\left(\mathrm{CC}_{50} \geq 58.5 \pm 4.6 \mu \mathrm{g} / \mathrm{ml}\right)$. An $\mathrm{EC}_{50}$ of $\leq 14.7 \pm 3.7 \mu \mathrm{g} / \mathrm{ml}$ for both wild type and resistant strains of HSV was realised in plaque and viral yield assays. Oral $(250 \mathrm{mg} / \mathrm{kg})$ and topical $(10 \%$ cream) administrations exhibited significant delay in onset of infections, hindered progression of infection to lethal forms with increased mean survival times and low mortality in both mice and guinea pig models. No acute toxicity has been realised at the therapeutic concentrations. TMR5 has demonstrated a high potential as an anti-herpes agent and arrangements are presently underway to evaluate its efficacy and safety in human clinical trials. A pilot production scheme supported by the National Commission for Science, Technology and Innovation (NCSTI) of Kenya has been undertaken as means of developing TMR5 as an alternative management therapy for herpes infections.

\section{P2.181 IL-4, IL-10 AND TNF-A PROFILE IN NORTH-EASTERN UKRAINIAN HIV-1 INFECTED INDIVIDUALS WITH DIFFERENT LEVEL OF IMMUNODEFICIENCY}

doi:10.1136/sextrans-2013-051184.0445

A Piddubna. Sumy State University, Sumy, Ukraine

Background Aim of the study was to determine the IL-4, IL-10 and TNF- $\alpha$ profiles in plasma of north-eastern Ukrainian HIV-1 infected individuals with different CD4 T-cell levels.

Methods We used a immunoassay method to measure IL-4, IL-10, TNF- $\alpha$ in plasma of 118 HIV-1 infected people among whom there were $80(67.8 \%)$ men and $38(32.2 \%)$ women aged (32.61 \pm 0.87$)$ years. Patients were divided into groups depending on the levels of CD4 T lymphocytes. Group I included 52 people with T-helper cell counts $\geq 350$ cells $/ \mu \mathrm{L}$, group II - 66 patients with T-helper cell counts $\leq 200$ cells $/ \mu \mathrm{L}$. Comparison group consisted of 30 normal healthy individuals.

Results In the cytokine profile of HIV-1 infected people the increased levels of pro-inflammatory cytokine TNF- $\alpha$ compared to controls (group I - $(0.77 \pm 0.08)$, group II - $(2.34 \pm 0.69)$, healthy controls - $(0.51 \pm 0.32) \mathrm{pg} / \mathrm{mL}, \mathrm{p}<0.05)$ and the anti-inflammatory IL-10 (group I - (3.99 \pm 0.99$)$, group II - $(20.08 \pm 0.44)$, healthy controls - $(1.68 \pm 0.32) \mathrm{pg} / \mathrm{mL}, \mathrm{p}<0.001)$ were demonstrated. No significant difference in IL-4 between surveyed troops and comparison group was found.

Patients with CD4 T lymphocyte levels $\leq 200$ cells/ $\mu \mathrm{L}$ showed significantly higher plasma concentration of TNF- $\alpha$ and IL-10 compared with the group I $(p<0.05)$. Among HIV-1 infected from group II mean serum concentrations of TNF- $\alpha$ higher than that of group I in 3 times $(p<0.05)$. A significant increase in the concentration of IL-10 detected in patients with severe immunodeficiency (IL-10 levels in group II was 5 times higher, $p<0.05$ ), which may indirectly indicate a more active involvement of IL-10 during disease progression.
Conclusion HIV-1 infection was associated with an increase in levels of TNF- $\alpha$ and IL-10. Immune imbalance due to changes in concentrations of cytokines is more pronounced in HIV-infected individuals with severe immunosuppression with CD4 $\mathrm{T}$ lymphocyte counts $\leq 200$ cells $/ \mu \mathrm{L}$.

\section{P2.182 MALE GENITAL DERMATOSES IN HIV}

doi:10.1136/sextrans-2013-051184.0446

${ }^{1} \mathbf{T}$ N Shim, ${ }^{2} \mathrm{D}$ Hawkins, ${ }^{2} \mathrm{~A}$ Muneer, ${ }^{1} \mathrm{~S}$ Minhas, ${ }^{1} \mathrm{~A}$ Freeman, ${ }^{1} \mathrm{C}$ Jameson, ${ }^{2} \mathrm{~N}$ Francis, ${ }^{2} \mathrm{M}$ Dinneen, ${ }^{1,2} \mathrm{C}$ B Bunker. ${ }^{1}$ University College Hospital London, London, UK; ${ }^{2} \mathrm{Chelsea}$ and Westminster Hospital, London, UK

Background Little information exists about penile squamous cell carcinoma (PSCC), penile carcinoma in situ (PCIS) and male genital lichen sclerosus (MGLSc) in HIV.

Methods A retrospective review of case notes was performed on HIV-positive men who had presented to specialised Male Genital Dermatoses Clinics between May 2011 and February 2013

Results 39 men were identified. The mean age at diagnosis was 48 years (range 26 - 71 years). The mean diagnostic delay was 20 months (range $1-72$ months). 35 were uncircumcised at presentation (4 were circumcised at birth/childhood). The majority of the cases had PCIS (21); 8 had MGLSc, 2 had lichen planus (GLP), 2 had PSCC and the remaining cases had more than one diagnosis (1 had PSCC and MGLSc, 2 had PSCC and PCIS, 3 had PCIS and MGLSc); 6 men had co-existing anal dysplasia (2 had anal SCC); 36 were on ARVs. All genital dermatoses were treated according to our conventional practise. The majority (31) is in remission; 6 have residual disease and receive ongoing treatment (1 GLP, 1 MGLSc and 4 PCIS); 2 have been lost to follow-up; most (32) have been circumcised (including 4 circumcised at birth/childhood). 1 has a short foreskin hence circumcision is not indicated.

Conclusion Advances in ARV treatment have improved the survival of individuals with HIV. This has led to increased interest in long-term morbidities, including cancer. MGLSc and PCIS can progress to invasive cancer. The risk of PSCC in HIV despite ARV treatemnt is $x 5-6$. The presence of the foreskin confers cancer risk. Uncircumcised HIV men should be counselled about the risk of PSCC. There may be an argument for prophylactic circumcision in high-risk cases such. Certainly, clinicians should enquire about the genital health of HIV-positive men and undertake regular (ano) genital examination at follow-up.

\section{P2.183 CLIMACTERIC SYMPTONS AND ASSOCIATED FACTORS IN HIV SEROPOSITIVE WOMEN}

doi:10.1136/sextrans-2013-051184.0447

A L Valadares, J Lui- Filho, L Costa-Paiva, E Amaral, M Souza, M Souza, A M PintoNeto. UNICAMP, Campinas - SP, Brazil

Background In the menopausal transition, the occurrence of insomnia, genito-urinary, psychological and.vasomotor symptoms may occur and this may be aggravated by the diagnosis of HIV infection.

Methods Cross-sectional study including 273 HIV-infected and 264 HIV uninfected Brazilian women. They were submitted to an interview to assess climacteric symptoms, socio-demographic characteristics and weight and height measurement.

Results The average age was $47.7 \pm 5.8$ years in HIV seropositive women and $49.8 \pm 5.3$ years in seronegative $(p<0.001)$. Bivariate analysis showed a lower prevalence of vasomotor symptoms in the HIV-positive Group $(p=0.009)$, specifically hot flushes $(p<0.002)$ and sweating $(p=0.049)$. Vaginal dryness was less prevalent in HIVpositive $(p<0.005)$. Depression and insomnia showed no statistical 
difference. Multiple analysis showed that hot flushes were associated with being in peri or postmenopause $(\mathrm{RP}=2.12,95 \% \mathrm{IC}=1.52$ $-2.94, p<0.001)$. The complaint of vaginal dryness was inversely associated with marital status $(\mathrm{RP}=0.67,95 \% \mathrm{CI}=0.49-0.90$, $\mathrm{p}=0.007)$, and directly associated with age $(\mathrm{RP}=1.03,95 \% \mathrm{IC}=1.01$ $-1.06, p=0.017)$ and being in peri-and postmenopause $(R P=1.69$, $95 \% \mathrm{IC}=1.10-2.60, \mathrm{p}=0.016)$. Depression was inversely associated with performing work $(\mathrm{RP}=0.74, \mathrm{CI}=95 \% 0.58-0.96, \mathrm{p}=0.022)$ and directly associated with the presence of chronic diseases $(\mathrm{RP}=1.30$, IC 95\% $=1.01-1.067, \mathrm{p}=0.043)$. Insomnia was associated to IMC (RP $=0.96$, CI 95\% $=0.95-0.97, \mathrm{p}<0.001)$ and peri-or postmenopausal ( $R P=1.48,95 \%$ CI $1.11-1.97, p=0.008)$. The serological status in relation to HIV has not been associated with any climacteric symptoms evaluated.

Conclusions HIV infection in these groups was not associated with vasomotor symptoms, genito-urinary, psychological, and insomnia.

\section{P2.184 FOLLOWING OF NEWLY MANAGED ADULT HIV INFECTED PATIENTS IN THE NATIONAL REFERRAL CENTRE, BURKINA FASO: THREE YEARS OUTCOMES STUDY}

\author{
doi:10.1136/sextrans-2013-051184.0448
}

'Y Sagna, ${ }^{1} \mathrm{H}$ Tiéno, ${ }^{1} \mathrm{O}$ Guira, 'I Diallo, ${ }^{1} \mathrm{E}$ A Diendéré, ${ }^{1,2} \mathrm{~J}$ Y Drabo. 'Internal Medicine department, Yalgado Ouédraogo University Hospital (CHU YO), Ouagadougou, Burkina Faso; ${ }^{2}$ African network of training on HIV (RAF-VIH), Ouagadougou, Burkina Faso

Background 10 years ago in Sub-Saharan countries, lab tests and antiretroviral therapy (ART) were expensive for most of patients. Many patients weren't able to start ART and those who start were frequently lost to follow-up. Since few years in Burkina-Faso, testing and treatment facilities are more available, ART is subsidised and starting when CD4 $<350$ cells $/ \mu$ l. We aimed to describe the present follow-up features among newly managed adult HIVinfected patients in this country national referral centre.

Methods a prospective and descriptive review was conducted, using a personal case report forms, in all newly managed adults with positive HIV antibody received between June and December 2009 at Yalgado Ouédraogo Hospital University Internal Medicine department in Ouagadougou. Each treated patient was followed-up for 36 months and had an appointment each 3 month the first year and each 6 month after with CD4 count and viral load measurement. All lab tests and ART were free of fees.

Results We received 191 patients with a median age of 37 (range, 18-65) years, sex ratio (M/W): 0.66. 177 patients returned to medical follow-up after the first consultation. 144/177 patients had CD4 $<350$ cells $/ \mu \mathrm{l}$ and have all been treated (first line). 128/144 treated patients were followed-up for the full 36 months and we have not recorded any major side effects of ART but the incidence of diabetes and dyslipidemia was respectively $2.3 \%$ and $1.6 \%$. 10 died and 7 were lost to follow up. The global compliance rate with appointments was $90 \%$. Weight gain and immune restoration were significant $(p<0.01)$. Two patients had a virological failure during the second year and they successfully switched to the second line treatment.

Conclusion we have excellent treatment success with no major side effects but we still lost to the follow-up some patients and mortality remains high.

\section{P2.185 HPV AND PREGNANCY: THE CONTROVERSIAL ROLE OF MATERNAL-FETAL TRANSMISSION}

doi:10.1136/sextrans-2013-051184.0449

${ }^{1}$ M Skerlev, ${ }^{2} \mathrm{M}$ Sirotković-Skerlev, 'S Ljubojević. 'Department of Dermatology and Venereology, Zagreb University Hospital and Zagreb University School of Medicine, Zagreb, Croatia; '2Department of Pathophysiology, Zagreb University School of Medicine, Zagreb, Croatia
Background and open questions Human papillomavirus (HPV) associated pathology represents one of the major problems among STIs mostly due to the high recurrence rate, difficult eradication and oncogenic potential. Young, sexually active population in the generative period is mostly affected. Pregnancy may definitely foster the development of latent or manifest HPV infections and may enhance the development of the pathology which then often recedes in the postpartum. The data reported in the literature on the relationship between HPV and pregnancy are highly controversial. A number of different opinions emerge regarding the possibility of maternal-fetal virus transmission. This discrepancy depends on the diagnostic techniques, the clinical history and the period of pregnancy when the sample is collected. The possible maternal-fetal transmission of the virus might be considered, for example, the main factor responsible for juvenile laryngeal papillomatosis, and the onset of the genital warts in children's age (apparently more often than the child's sexual abuse which should not be neglected either!). Many authors report an initial presence of HPV in newborns which often disappears within 6 months after birth.

Conclusion According to the most recent vaccination recommendations, HPV vaccines are not recommended for use in pregnant women. However, pregnancy testing is not needed before vaccination. If a woman is found to be pregnant after initiating the vaccination series, no intervention is needed; the remainder of the 3-dose series should be delayed until completion of pregnancy. Providing the controversies that still exist, modes of HPV transmission in pregnancy should be investigated more detailfully in order to get clearer picture and a fuller awareness of the preventive measures. It can be concluded that, in this very moment, there is a need for a coordinated effort of health professionals and policymakers to ensure successful implementation of vaccination programmes for both women and men.

\section{P2.186 MANAGEMENT OF GENITAL HPV INFECTION IN MEN}

doi:10.1136/sextrans-2013-051184.0450

${ }^{1} \mathrm{~F}$ Sandru, ${ }^{2} \mathrm{~A}$ V Dragodan, ${ }^{1} \mathrm{M}$ C Dumitrascu, ${ }^{3} \mathrm{G}$ Turcu, ${ }^{3}$ D Forsea..$~ "$ Euromed" Medical Center, Bucharest, Romania; " $"$ Stefan S Nicolau" Institute of Virology, Bucharest, Romania; ${ }^{3 " C}$ arol Davila" University of Medicine and Pharmacy, Bucharest, Romania

Background HPVgenital infections is not yet a STD to be reported. The actual incidence and prevalence are increasing and should be updated using new molecular biology techniques. In men it is more difficult to establish prevalence because of difficult sampling of a suitable biological product.

Methods The study involved the participation of 105 men (20062011), aged 18-55 years, selected from patients with external anogenital lesions with HPV suspected aetiology or who had partner/ sexual partners with clinical lesions and/or positive test for HPV. The tests used for HPV DNA detection and genotyping were: HPV INNO - Lipa HPV genotyping Line Probe Assay EC (Innogenetics) and LINEAR ARRAY HPV genotyping test (Roche Diagnostics).

Results HPV distribution and the genotypes most commonly found were: in men without clinical signs - 26 cases $(24.76 \%) / \mathrm{HPV}$ $18,45,6,11,16,31,33,61,66$, CP6108, and those with acuminate and external genital flat warts -16 cases $(15.23 \%) / \mathrm{HPV} 6,11, \mathrm{X}$ (indeterminate), 40, 45, 55 - single or multiple infection.

The applied treatment was: Inosinum PO for porting cases, Inosinum $\mathrm{PO}$ and $\mathrm{CO}_{2}$ laser vaporisation for those with external anogenital warts acuminata 13 cases $(12.38 \%$ ), or Inosinum PO and local podophyllotoxin $0.5 \%$ for those with external genital warts flat 3 cases $(2.85 \%)$.

Conclusion Proper counselling of symptomatic or asymptomatic male patients infected with HPV, together with using all investigative protocols and personalised therapy, provides, in $90 \%$ of cases, healing of external genital lesions induced by HPV virus and negative results for HPV tests. 